\title{
Relationship of Lifestyle Medical Advice and Non-HDL Cholesterol Control of a Nationally Representative US Sample with Hypercholesterolemia by Race/Ethnicity
}

\author{
Joan Anne Vaccaro ${ }^{1}$ and Fatma G. Huffman ${ }^{2}$ \\ ${ }^{1}$ Department of Dietetics and Nutrition, MMC AHC 1-450, Florida International University, Miami, FL 33199, USA \\ ${ }^{2}$ Department of Dietetics and Nutrition, MMC AHC 1-435, Florida International University, Miami, FL 33199, USA
}

Correspondence should be addressed to Fatma G. Huffman, huffmanf@fiu.edu

Received 16 June 2012; Revised 3 August 2012; Accepted 16 September 2012

Academic Editor: Gloria L. Vega

Copyright (C) 2012 J. A. Vaccaro and F. G. Huffman. This is an open access article distributed under the Creative Commons Attribution License, which permits unrestricted use, distribution, and reproduction in any medium, provided the original work is properly cited.

\begin{abstract}
Objective. The main purpose of this study was to evaluate the associations of lifestyle medical advice and non-HDL cholesterol control of a nationally representative US sample of adults with hypercholesterolemia by race/ethnicity. Methods. Data were collected by appending sociodemographic, anthropometric, and laboratory data from two cycles of the National Health and Nutrition Survey (2007-2008 and 2009-2010). This study acquired data from male and female adults aged $\geq 20$ years $(N=11,577)$, classified as either Mexican American (MA), $(n=2173)$, other Hispanic $(\mathrm{OH})(n=1298)$, Black non-Hispanic (BNH) $(n=2349)$, or White non-Hispanic (WNH) $(n=5737)$. Results. Minorities were more likely to report having received dietary, weight management, and exercise recommendations by healthcare professionals than WNH, adjusting for confounders. Approximately $80 \%$ of those receiving medical advice followed the recommendation, regardless of race/ethnicity. Of those who received medical advice, reporting "currently controlling or losing weight" was associated with lower non-HDL cholesterol. BNH who reported "currently controlling or losing weight" had higher non-HDL cholesterol than WNH who reported following the advice. Conclusion. The results suggest that current methods of communicating lifestyle advice may not be adequate across race/ethnicity and that a change in perspective and delivery of medical recommendations for persons with hypercholesterolemia is needed.
\end{abstract}

\section{Introduction}

Cholesterol, the functional unit of numerous, essential hormones and steroids in the human body, circulates in blood. Even though cholesterol is necessary for body function, elevated levels can result in atherosclerosis and cardiovascular disease. Levels of serum cholesterol less than $200 \mathrm{mg} / \mathrm{dL}$ are considered in the healthy, normal range; borderline cholesterol is $200-239 \mathrm{mg} / \mathrm{dL}$; elevated blood cholesterol, $240 \mathrm{mg} / \mathrm{dL}$ or above, is classified as high cholesterol [1]. Primary goals of therapy and treatment are focused on low-density lipoprotein cholesterol (LDLC) $<100 \mathrm{mg} / \mathrm{dL}$, where $130 \mathrm{mg} / \mathrm{dL}$ is considered borderline high [1]. Despite the decrease in LDL-C since the 1960s, hypercholesterolemia, a key risk factor of atherosclerosis and coronary heart disease, currently affects nearly half of the US adult population [2]. Hypercholesterolemia is a metabolic disorder characterized by high levels of serum cholesterol, particularly LDL-C. For treatment purposes, hypercholesterolemia individually diagnosed based on high LDL-C and concurrence of other cardiovascular disease risk factors such as smoking, hypertension, diabetes, and a family history of premature coronary heart disease [3]. The recommended treatment of hypercholesterolemia involves weight loss, dietary and physical activity changes, and a possible medical regime. Prescription of LDL-C lowering medications should be reserved for aggressive treatment when LDL-C levels do not respond to dietary modifications and increased exercise $[3,4]$. The side effects of statins, a commonly prescribed cholesterol-lowing medication, may outweigh their benefit for otherwise healthy adults [4]. 
A diagnosis of hypercholesterolemia and consequent treatment is only possible for adults who had adequate medical care, which included blood collection for a lipid panel. Among a nationally representative sample of US adults in 2005-2006, aged 20 years or older, only three-quarters reported ever having their cholesterol checked with lower reports for Mexican Americans and non-Hispanic Blacks as compared to non-Hispanic Whites [2]. Minorities have been reported to have less access and quality of health care for cholesterol screening and treatment [5]. Even when treated for hypercholesterolemia, a lower percent of minorities were prescribed dietary and exercise counseling, based on a representative US sample of approximately 27 million adults, aged $\geq 20$ years, from a medical registry [5].

Because the initial treatment of hypercholesterolemia involves dietary and physical activity changes, it is imperative that the diagnosed individuals recall having received these lifestyle medical recommendations. Therefore, the aim of this study was to examine a US representative sample of adults for the years 2007-2010 and to investigate the following (1) the likelihood of lifestyle medical advice received differing by race/ethnicity for those adults who had their cholesterol checked; (2) percents of adults given and following medical advice; (3) the association of non-HDL cholesterol with reporting following lifestyle medical advice.

\section{Materials and Methods}

2.1. Data Collection. All data used for this study were approved by the research ethics board and publically available from appended 2-year cycles of datasets from the National Health and Nutrition Examination Survey (NHANES) 2007-2008 and 2009-2010 [6]. NHANES uses a complex, multistage, probability sample design to obtain representative samples of the noninstitutionalized, civilian US population [6]. This study acquired data from male and female adults aged $\geq 20$ years $(N=11,577)$. The final sample size for participants with hypercholesterolemia and measurements of total and/or non-HDL cholesterol was 3325 $(\mathrm{MA}=505 ; \mathrm{OH}=362 ; \mathrm{BNH}=614 ; \mathrm{WNH}=1844)$.

2.2. Major Variables. Hypercholesterolemia was considered an affirmative answer to "ever told by a doctor or other health care professional that you had a high blood cholesterol level". Non-HDL cholesterol was constructed by subtracting HDLC from total cholesterol. Cholesterol control (yes/no) was constructed as a binary variable with non-HDL cholesterol $<130 \mathrm{mg} / \mathrm{dL}$ considered adequate control versus $\geq 130 \mathrm{mg} / \mathrm{dL}$ as inadequate control. The number of participants with this cutoff was chosen based on the National Cholesterol Education Program's (NCEP-ATP III) guideline of $130 \mathrm{mg} / \mathrm{dL}$ for persons with $>20 \%$ cardiovascular disease risk [1] and the American Association of Clinical Endocrinologists' (AACE) guidelines of non-HDL cholesterol as $30 \mathrm{mg} / \mathrm{dL}$ above the goal $(<100 \mathrm{mg} / \mathrm{dL})$ for LDL-C [7]. Medical advice (yes, no) for weight, diet, and exercise were the major independent variables and phrased as follows: to lower your blood cholesterol have you ever been told by your doctor or other health professional to: (1) eat fewer high-fat or high-cholesterol foods? (2) control weight or lose weight? (3) increase physical activity or exercise?

2.3. Adjustment Variables. Comorbidities of hypercholesterolemia used in the analysis were coronary heart disease, diabetes, and hypertension. Coronary heart disease was defined as an affirmative response to the question, "Has a doctor or other health professional ever told you that you had coronary heart disease?" Diabetes was considered an affirmative answer to the question, "Has a doctor or other health care professional told you that you had diabetes or sugar diabetes?" Having hypertension was defined as a systolic blood pressure $\geq 140 \mathrm{~mm} \mathrm{Hg}$, a diastolic blood pressure $\geq 90 \mathrm{~mm} \mathrm{Hg}$ (using the average of first two readings), or taking hypertension medication [8].

Obesity was estimated using the proxy measurement of body mass index (BMI) and was calculated from measured weight and height $\left(\mathrm{kg} / \mathrm{m}^{2}\right)$ and was categorized based on the National Institutes of Health's recommended levels: normal weight $\left(<24.9 \mathrm{~kg} / \mathrm{m}^{2}\right)$; overweight $\left(24.9-29.9 \mathrm{~kg} / \mathrm{m}^{2}\right)$; obese I $\left(30.0-34.4 \mathrm{~kg} / \mathrm{m}^{2}\right)$; obese II or more $\left(\geq 35 \mathrm{~kg} / \mathrm{m}^{2}\right)$ [9]. A binary variable was created for current cigarette use versus not currently smoking. Education was assessed as $<$ high school; high school diploma or general equivalency diploma; $>$ high school. Health insurance was defined as having any coverage within the past 12 months (yes/no).

2.4. Statistical Analysis. Chi-square tests were performed to determine hypercholesterolemia and medical advice by ethnicity. Logistic regression analyses were conducted for non-HDL cholesterol control as the outcome variable. Reduced models included race/ethnicity adjusted for age and gender. Fully adjusted models included the covariates of the simple models along with: currently smoking, BMI, education, health insurance, and lipid-lowering medication. In addition, hypertension, diabetes, and coronary heart disease were added to test medical advice received by race/ethnicity. Adjusted sample weights were used to account for unequal probabilities of selection and nonresponse in the multistage stratified cluster sampling design used in NHANES in order to achieve unbiased national estimates. The sample weights used were based on the data file with the smallest sample size, the Mobile Examination Center (MEC), and were computed using the average of the 2-year sample weights for each cycle, as per guidelines set by NHANES [10]. Data analysis was conducted with complex module, IBMSPSS version 19 . The Wald $F$ statistic was used to determine model significance for logistic regression analysis [11]. For the chi-squared test, significance was based on the adjusted $F$ statistic, a variant of the second-order Rao Scott adjusted chisquare statistic used for analyses with sample weights, where $\mathrm{df} 1+\mathrm{df} 2=33$ (total degrees of freedom).

\section{Results}

Key risk factors associated with hypercholesterolemia are shown in Table 1. There were significant differences in major 
TABLE 1: Major hypercholesterolemia risk factors of the study population by race/ethnicity.

\begin{tabular}{|c|c|c|c|c|c|}
\hline Variable & MA & $\mathrm{OH}$ & $\mathrm{BNH}$ & WNH & $P$ value \\
\hline Cholesterol checked (yes) & $51.2(1.6)^{\mathrm{a}}$ & $60.5(2.2)^{\mathrm{b}}$ & $69.7(1.3)^{\mathrm{c}}$ & $78.5(0.9)^{\mathrm{d}}$ & $<.001$ \\
\hline Inadequate non-HDL-C ( $\geq 130 \mathrm{mg} / \mathrm{dL})$ & $25.8(2.0)^{\mathrm{a}}$ & $22.1(2.5)^{\mathrm{a}}$ & $33.1(2.1)^{\mathrm{b}}$ & $30.6(1.1)^{\mathrm{b}}$ & .008 \\
\hline High cholesterol ( $\geq 200 \mathrm{mg} / \mathrm{dL})$ & $45.9(1.4)^{\mathrm{a}}$ & $42.1(2.2)^{\mathrm{a}, \mathrm{b}}$ & $39.7(1.3)^{\mathrm{b}}$ & $44.5(0.9)^{\mathrm{a}, \mathrm{b}}$ & .019 \\
\hline Lipid-lowering medications (yes) & $50.7(3.4)^{-}$ & $44.1(3.4)^{-}$ & $51.7(1.8)^{-}$ & $54.7(1.6)^{-}$ & .129 \\
\hline Age (years) & $51.2(1.2)^{\mathrm{a}}$ & $52.3(1.1)^{\mathrm{a}, \mathrm{b}}$ & $53.8(0.5)^{\mathrm{b}}$ & $57.4(0.3)^{\mathrm{c}}$ & $<.001$ \\
\hline Education: $<$ HS & $51.5(1.6)^{\mathrm{a}}$ & $37.7(2.4)^{\mathrm{b}}$ & $26.1(1.7)^{\mathrm{c}}$ & $13.8(1.4)^{\mathrm{d}}$ & $<.001$ \\
\hline No health insurance & $52.2(1.9)^{\mathrm{a}}$ & $36.3(3.1)^{\mathrm{b}}$ & $26.0(1.4)^{\mathrm{c}}$ & $13.7(0.7)^{\mathrm{d}}$ & $<.001$ \\
\hline Current smoker & $11.4(1.1)^{\mathrm{a}}$ & $14.5(1.8)^{\mathrm{a}}$ & $21.7(1.7)^{\mathrm{b}}$ & $19.1(1.2)^{\mathrm{b}}$ & $<.001$ \\
\hline BMI 30-34.9 $\left(\mathrm{kg} / \mathrm{m}^{2}\right)$ obesity I & $22.5(1.6)^{\mathrm{a}}$ & $18.6(1.4)^{\mathrm{b}, \mathrm{c}}$ & $21.6(1.3)^{\mathrm{a}, \mathrm{c}}$ & $18.5(0.6)^{\mathrm{b}}$ & $<.001$ \\
\hline $\mathrm{BMI} \geq 35\left(\mathrm{~kg} / \mathrm{m}^{2}\right)$ obesity II & $18.0(0.8)^{\mathrm{a}}$ & $17.0(1.3)^{\mathrm{a}}$ & $26.1(1.2)^{\mathrm{b}}$ & $16.2(0.6)^{\mathrm{a}}$ & $<.001$ \\
\hline HTN (yes) & $19.2(1.4)^{\mathrm{a}}$ & $21.1(1.6)^{\mathrm{a}}$ & $38.7(1.3)^{\mathrm{b}}$ & $31.8(1.0)^{\mathrm{c}}$ & $<.001$ \\
\hline Diabetes (yes) & $8.4(0.8)^{\mathrm{a}}$ & $8.5(0.8)^{\mathrm{a}}$ & $13.5(0.8)^{\mathrm{b}}$ & $7.6(0.6)^{\mathrm{c}}$ & $<.001$ \\
\hline CHD (yes) & $1.8(0.3)^{\mathrm{a}}$ & $1.4(0.3)^{\mathrm{a}}$ & $1.6(0.2)^{\mathrm{a}}$ & $3.7(0.2)^{\mathrm{b}}$ & $<.001$ \\
\hline
\end{tabular}

MA: Mexican American; OH: other Hispanic, not Mexican; BNH: Black non-Hispanic; WNH: White non-Hispanic; non-HDL-C: non-HDL cholesterol (total cholesterol minus HDL-C); BMI: body mass index; HTN: hypertension; CHD: coronary heart disease.

The data represent the weighted percent and standard error [\% (SE)]. Significance is based on the adjusted F, a variant of the second-order Rao-Scott adjusted chi-square statistic and its degrees of freedom. Each superscript letter denotes a subset of race/ethnicity categories whose column proportions do not differ significantly from each other at the 0.05 level for each row (variable). Significant differences between each group were measured by complex logistic regression analyses across race/ethnicity separately for each dependent binary variable. Age was tested by complex general linear models, recoding ethnicity to measure differences between groups.

sociodemographics, body mass index, morbidities, and having cholesterol checks among race/ethnicity. WNH had the highest prevalence of reporting having their cholesterol checked followed by $\mathrm{BNH}, \mathrm{OH}$, and MA. For those with hypercholesterolemia, coronary heart disease was higher in WNH than other race/ethnicity categories. $\mathrm{BNH}$, followed by $\mathrm{WNH}$, had higher prevalence of hypertension as compared to Hispanics. BNH had a higher prevalence of obesity II $\left[B M I \geq 35\left(\mathrm{~kg} / \mathrm{m}^{2}\right)\right]$ and diabetes than other groups. A lower percent of minorities were covered by health insurance with MA having the least coverage.

The likelihood of receiving medical advice by race/ethnicity is shown in Table 2. Compared to $\mathrm{WNH}$, $\mathrm{MA}$ and $\mathrm{BNH}$ were more likely to be given advice to eat fewer high-fat or -cholesterol foods. MA and $\mathrm{OH}$ were more likely to be told to lose or control their weight as compared to WNH. All Hispanics and BNH were more likely to be told to increase physical activity or exercise as compared to $\mathrm{WNH}$.

The percent of adults with hypercholesterolemia who reported affirmatively that they were given diet, weight, and exercise medical advice to reduce their cholesterol levels and who reported affirmatively now following the advice is shown in Table 3. Despite the fact that weight management and increasing physical activity are vital recommendations for individuals with hypercholesterolemia, approximately $40 \%$ and $30 \%$, respectively, reported not receiving advice for these lifestyle behaviors. The majority of the participants (approximately 80\%), who reported having received medical advice, followed the corresponding recommendations.

Participants who responded affirmatively to being given medical advice for hypercholesterolemia were included in a chi-squared test of whether they followed each recommendation (yes/no) across non-HDL-C control $(<130 \mathrm{mg} / \mathrm{dL}$, yes $/ \geq 130 \mathrm{mg} / \mathrm{dL}$, no) (Table 3 ). Reporting now eating fewer fatty foods $(P=.002)$ and now losing weight $(P=.005)$ were significantly associated with the corresponding recommendation. Cholesterol control (non-HDL-C $<130 \mathrm{mg} / \mathrm{dL}$ ) was found for approximately two-thirds of those who reported eating fewer fatty foods $(67.5 \%, \mathrm{SE}=1.2 \%)$. Similarly, of those participants who reported now controlling or losing weight, $67.3 \%(\mathrm{SE}=1.4 \%)$ had cholesterol control (nonHDL-C $<130 \mathrm{mg} / \mathrm{dL}$ ).

Reporting following medical recommendations for diet and physical activity (yes/no) and non-HDL cholesterol (continuous) was tested by a complex general linear model. The model was significant; however, of the behaviors only "now controlling or losing weight" was significant (data not shown). Reduced and full models were constructed for reporting "controlling or losing weight" by race/ethnicity (Table 4). Lower non-HDL cholesterol levels were associated with an affirmative report of "now controlling or losing weight" for the reduced model (adjusted only for age and gender $(P=.001))$. For the fully adjusted model, which included, BMI, health insurance, education, currently smoking, and lipid-lowering medication(s), the 2-way interaction "controlling or losing weight" by race/ethnicity was significant $(P=.014)$ and the main effect (controlling or losing weight) was no longer significant $(P=.998)$; however race/ethnicity remained significant $(P<.001)$. BNH who reported "controlling or losing weight" had significantly higher non-HDL cholesterol as compared to WNH $(P=$ $.003)$.

Use of lipid-lowering medications did not negate the association of losing weight with non-HDL cholesterol as a continuous variable; however, for logistic regression models lipid-lowering medications did negate the likelihood of having cholesterol control (non-HDL cholesterol $<130 \mathrm{mg} / \mathrm{dL}$ ); albeit the results were marginal $[\mathrm{OR}=1.49(1.18,1.76)$, 
TABLE 2: Odds ratios for receiving medical advice by race/ethnicity.

\begin{tabular}{lcccc}
\hline Dependent variable & Independent & Model 1 & Model 2 & Model 3 \\
\hline & MA & $1.48(1.09,2.04)$ & $1.59(1.14,2.20)$ & $1.60(1.14,2.26)$ \\
Told to eat less fat & OH & $1.10(0.81,1.48)$ & $1.13(0.82,1.56)$ & $1.15(0.82,1.60)$ \\
& BNH & $1.70(1.36,2.12)$ & $1.59(1.37,1.99)$ & $1.49(1.19,1.86)$ \\
& WNH & 1.00 & 1.00 & 1.00 \\
Told to control or lose weight & MA & $1.54(1.12,2.12)$ & $1.58(1.12,2.21)$ & $1.55(1.07,2.25)$ \\
& OH & $1.75(1.33,2.31)$ & $1.37(1.32,2.39)$ & $1.75(1.26,2.41)$ \\
& BNH & $1.37(1.11,1.70)$ & $1.1,1.71)$ & $1.21(0.95,1.54)$ \\
Told to exercise & WNH & 1.00 & $1.64(1.21,2.23)$ & 1.00 \\
& MA & $1.65(2.23,2.22)$ & $1.56(1.19,2.06)$ & $1.54(1.15,2.07)$ \\
& OH & $1.56(1.20,2.04)$ & $1.51(1.24,1.85)$ & $1.35(1.09,1.68)$ \\
\hline
\end{tabular}

Model 1: adjusted for age and gender (and BMI for "told to control or lose weight").

Model 2: adjusted for age, gender, BMI, current smoker, education, and health insurance.

Model 3: adjusted for age, gender, BMI, current smoker, education, health insurance, coronary heart disease, diabetes, and hypertension.

TABLE 3: Percent of adults given and following medical advice.

\begin{tabular}{lll}
\hline Given the recommendation to lower serum cholesterol & $\begin{array}{l}\text { Percent given the advice who are } \\
\text { following the recommendation }\end{array}$ & SE \\
\hline Told to eat less high-fat or high-cholesterol foods $81.8 \%$ (yes) & $82.8(80.7,84.7)$ & 1.0 \\
Told to control weight or lose weight $59.4 \%$ (yes) & $84.0(81.3,86.3)$ & 1.2 \\
Told to increase physical activity or exercise $69.9 \%$ (yes) & $78.0(75.4,80.3)$ & 1.2 \\
\hline
\end{tabular}

Results are given as [mean $(95 \% \mathrm{CI}) \mathrm{SE}]$.

with independents: race, gender, age, BMI, health insurance, education, and smoking; $\mathrm{OR}=1.18(0.93,1.49)$ with the same independents and the additional covariate, lipidlowering medications] (data not shown).

\section{Discussion}

In a nationally representative population of adults with hypercholesterolemia, we found discrepancies in medical advice for diet, weight management, and exercise by race/ethnicity. Reporting having received medical advice was associated with performing the recommended behavior; yet, over one-third of adults across race/ethnicity with hypercholesterolemia reported not receiving dietary, weight management, or exercise recommendations by either their physician or other health care professional. Moreover, there were differences by race/ethnicity in reporting having received these lifestyle recommendations. These results suggest confounders such as patient-provider relationships and health beliefs, which may vary by race/ethnicity and may also influence interpretation of advice and resulting health outcomes.

African Americans, as compared to $\mathrm{WNH}$ with comparable healthcare coverage, were found to have a poorer quality of patient-provider relationships as well as poorer control of cholesterol, in a national, multicentered prospective study of patients with diabetes [12]. In the current study, $\mathrm{BNH}$ who reported "controlling or losing weight" had significantly higher non-HDL cholesterol as compared to WNH. This disparity may be the result of differences in perceived vulnerability or susceptibility by race/ethnicity. In this case, $\mathrm{BNH}$ may not perceive danger of cardiac events until their cholesterol level in a higher range than their $\mathrm{WNH}$ counterparts.

The Agency for Health Care Research and Quality (AHRQ) [13] calculated 70\% disparity (worsening or no improvement) between minorities and $\mathrm{WNH}$ with respect to quality of care change in five years (from 2003 to 2008). In contrast to the findings of Willson et al. [5], minorities were more likely to receive medical advice than WNH in our analysis; however, patient-provider relationships were not assessed and may have confounded the results. It has been suggested that physicians who positively engage minority patients may either be members of a minority group or have had experiences dealing with minority patient's health barriers and may be acting in an attempt to reduce health disparities [14]. Piette et al. [15] found that African Americans and Spanish-speaking patients as well as patients with a lower education reported having better communication with their physicians than patients of other race/ethnicities. Conversely, African American patients reported poorer patient-physician communication as compared to Whites with the same healthcare coverage in a national multicenter prospective study [12]. The investigators found that health outcomes and disease control had been positively associated with the quality of patient-physician communication, as measured by the patients' trust and report of physicians listening, explaining, showing respect, and spending time [12]. 
TABLE 4: Effect of reporting "now controlling or losing weight" on non-HDL cholesterol of those participants who were told to "control or reduce weight" by race/ethnicity.

\begin{tabular}{|c|c|c|c|c|}
\hline \multirow{2}{*}{ Independent variables } & \multicolumn{2}{|c|}{ Model 1} & \multicolumn{2}{|c|}{ Model 2} \\
\hline & $\mathrm{B}(95 \% \mathrm{CI})$ & SE & $\mathrm{B}(95 \% \mathrm{CI})$ & SE \\
\hline Now controlling or losing weight (yes) & $-8.9(-13,-4.5)$ & $2.2^{*}$ & $-4.4(-8.4,-1.3)$ & 1.8 \\
\hline MA & $2.2(-5.8,10.2)$ & 3.9 & $-1.1(-9.1,6.8)$ & 3.9 \\
\hline $\mathrm{OH}$ & $-2.9(-12,6.5)$ & 4.7 & $-3.8(-12,4.6)$ & 4.1 \\
\hline $\mathrm{BNH}$ & $-16(-22,-11)$ & $2.8^{*}$ & $-18(24,11)$ & $3.2^{*}$ \\
\hline Lose wt by MA & $0.70(-9.9,11)$ & 5.2 & $0.66(-8.2,9,5)$ & 4.3 \\
\hline Lose wt by $\mathrm{OH}$ & $9.7(-0.32,19)$ & 4.9 & $5.0(-4.0,14)$ & 4.4 \\
\hline Lose wt by BNH & $15(6.8,23)$ & $4.1^{*}$ & $14(5.2,22)$ & $4.2^{*}$ \\
\hline \multirow{2}{*}{ Model summary } & \multicolumn{2}{|c|}{$R^{2}=0.063$} & \multicolumn{2}{|c|}{$R^{2}=0.21$} \\
\hline & \multicolumn{2}{|c|}{$F(9,24)=41.0, P<0.001$} & \multicolumn{2}{|c|}{$F(17,16)=58.7, P<0.001$} \\
\hline
\end{tabular}

Non-HDL cholesterol: non-high-density lipoprotein cholesterol; MA: Mexican American; OH: other Hispanic; BNH: Black, non-Hispanic; wt: weight. Model 1 was adjusted for gender and age. Model 2 included body mass index, health insurance, education, currently smoking, and taking cholesterol-lowering medication(s) along with the adjustments of Model 1 . White non-Hispanic (WNH) is the reference group for race/ethnicity. Significance $(P<0.05)$ is denoted by $(*)$.

In the current study, there were no differences in LDLC managing behaviors among race/ethnicity for adults with hypercholesterolemia given lifestyle medical advice. Instead, being given medical recommendations was associated with making the changes. These findings support the benefit of secondary prevention measures of diet and exercise recommendations by medical professionals. Several studies found racial/ethnic differences in therapeutic lifestyle changes for patients with chronic disease. BeLue et al. [16] reported different patterns of diet-exercise behavior (fruit and vegetable intake, fat intake and exercise) by race/gender groups for Black and White adults with hypercholesterolemia. Oster et al. [17] reported that Blacks and Hispanics were less likely to monitor their diet than WNH with diabetes in a national managed care organization. Moreover, for patients with diabetes, Blacks were less likely to exercise as compared to Whites $[17,18]$.

Weight loss, but not reducing fatty foods nor increasing physical activity, was associated with lower non-HDL cholesterol in our study. Several meta-analyses of dietary interventions for cardiovascular risk reduction have reported modest effects in cholesterol reduction [19-21]. Albeit, even modest reductions in serum cholesterol, about $10 \%$, have been associated with a $50 \%$ decrease in the incidence of coronary heart disease for age 40 and $20 \%$ for age 70 for approximately 500,000 men in a meta-analysis of cohort studies [22].

In order for serum non-HDL cholesterol management to be effective, several precursors are necessary: (1) access to quality medical care that provides a diagnosis of hypercholesterolemia; (2) a culturally appropriate, nondiscriminatory environment that encourages continuation of healthcare utilization; and, (3) effective patient-provider relationships that involve patients in their treatment plans and empower them to follow disease-specific recommendations. Barriers for receiving and interpreting medical advice may differ by race/ethnicity. Based on a national survey of healthcare quality, African Americans, Hispanics, and Asians reported more perceived healthcare provider discrimination than $\mathrm{WNH}$
[23]. Poorer health status was associated with perceived healthcare discrimination more frequently in minorities than WNH [23]. There is inconclusive evidence regarding differences in patient-provider communication among the Hispanic subgroups. Although access to healthcare varies among Hispanic groups [24], the differences in access were not related to the quality of communication between the patient and their healthcare provider [25].

Receiving advice may not be interpreted equally across patients and may be a confounder in serum non-HDL cholesterol control. In particular, the interpretation of the medical recommendation to "eat fewer high-fat or highcholesterol foods" may not be consistent among patients and may vary by race/ethnicity. Since people usually eat $a d$ libitum (until they are satisfied), they are likely to exchange one food for another, rather than to reduce total calories [26]. In an effort to reduce "fatty foods," patients may substitute any combination of the following: refined carbohydrates (such as white flour or white rice), unrefined grains (such as whole oats, wheat, or brown rice), or healthy fats (such as nuts, seeds, avocados, and mono- and poly-unsaturated oils) for "fatty foods." The range of actions resulting in the interpretation of this dietary advice may have been a confounder in non-HDL cholesterol control.

Numerous prospective studies of both primary and secondary preventive measures to reduce the prevalence of hypercholesterolemia suggest that these efforts may prove to be beneficial by reducing its prevalence in the US. Healthy adults followed for 27 years were $26 \%$ and $30 \%$ less likely to develop hypercholesterolemia that maintained or increased their fitness level, respectively, compared to those who lost fitness levels [27]. Secondary prevention, aimed at non-HDL cholesterol control for those with hypercholesterolemia, also entails medical recommendations for diet, weight, and physical activity; yet, research regarding the efficacy of advice with behavior changes and health outcomes for persons with hypercholesterolemia is limited.

Our results should be interpreted with respect to the study strengths and limitations. A major strength of this 
study was the use of a large, nationally representative dataset. Another strength of this study was the use of non-HDL cholesterol as both a binary and continuous variable to measure cholesterol control as opposed to total cholesterol. The use of non-HDL cholesterol $<130 \mathrm{mg} / \mathrm{dL}$ as adequate control may not be a suitable goal for all persons with hypercholesterolemia. The AACE guidelines are that goals for non-HDL cholesterol be set at $30 \mathrm{mg} / \mathrm{dL}$ above the target for LDL-C [7]. Although a target of non-HDL cholesterol $<130 \mathrm{mg} / \mathrm{dL}$ is recommended for most patients, guidelines for lipid goals for patients at risk for coronary artery disease vary by physician and should be set as $30 \mathrm{mg} / \mathrm{dL}$ above the patient's LDL-C goals [7]. Several limitations were noted. The category "Others" was not used due to a small sample size. Data concerning having serum cholesterol checked and being given medical advice to manage hypercholesterolemia were self-reported and may contain some inaccuracies. Moreover, bias by race/ethnicity, age, gender, or other characteristics are possible due to recall differences among people.

\section{Conclusions}

The findings of this study indicate a lack of congruence in following medical advice and achieving adequate non-HDL cholesterol control by race/ethnicity. These results suggest that current methods of communicating lifestyle advice may not be adequate across ethnicity/race and that a change in perspective and delivery of medical recommendations for persons with hypercholesterolemia is needed. Evaluation of patient-provider communication with cholesterol management across race/ethnicity may be necessary to develop effective dietary and physical activity intervention strategies. The results of our study may inform healthcare professionals of the need to develop client-centered treatment plans with clear, individualized goals for diet, weight management, and physical activity.

\section{Conflict of Interests}

The authors declare that they have no conflict of interests.

\section{References}

[1] National Cholesterol Education Program, ATP III. US Department of Health and Human Services, Public Health Service National Institutes of Health National Heart, Lung, and Blood Institute. NIH Publication No. 01-3305, 2012 http://www .nhlbi.nih.gov/guidelines/cholesterol.

[2] E. S. Ford, C. Li, W. S. Pearson, G. Zhao, and A. H. Mokdad, "Trends in hypercholesterolemia, treatment and control among United States adults," International Journal of Cardiology, vol. 140, no. 2, pp. 226-235, 2010.

[3] "Third Report of the National Cholesterol Education Program (NCEP) Expert Panel on Detection, Evaluation, and Treatment of High Blood Cholesterol in Adults (Adult Treatment Panel III) final report," Circulation, vol. 106, no. 25, pp. 31433421, 2002.

[4] R. F. Redberg and M. H. Katz, "Healthy men should not take statins," JAMA, vol. 307, no. 14, pp. 1491-1492, 2012.
[5] M. N. Willson, J. J. Neumiller, D. A. Sclar, L. M. Robison, and T. L. Skaer, "Ethnicity/Race, use of pharmacotherapy, scope of physician-ordered cholesterol screening, and provision of diet/nutrition or exercise counseling during us office-based visits by patients with hyperlipidemia," American Journal of Cardiovascular Drugs, vol. 10, no. 2, pp. 105-108, 2010.

[6] National Health and Nutrition Examination Survey, Questionnaires, datasets and related documentation, http://www .cdc.gov/nchs/nhanes/nhanes_questionnaires.htm.

[7] P. S. Jellinger, D. A. Smith, A. E. Mehta et al., "American association of clinical endocrinologists' guidelines for management of dyslipidemia and prevention of atherosclerosis," Endocrine Practice, vol. 18, supplement 1, pp. 1-78, 2012.

[8] A. V. Chobanian, G. L. Bakris, H. R. Black et al., "Seventh report of the Joint National Committee on Prevention, Detection, Evaluation, and Treatment of High Blood Pressure," Hypertension, vol. 42, no. 6, pp. 1206-1252, 2003, http:// www.nhlbi.nih.gov/guidelines/hypertension/index.htm.

[9] National Institutes of Health, National Heart, Lung and Blood Institute. NHLBI Obesity education initiative expert panel on the identification, evaluation, and treatment of overweight and obesity in adult. NIH publication 00 4084, October 2000.

[10] National Center for Health Statistics (NCHS): Center for Disease Control and Prevention. National Health and Nutrition Examination Survey (NHANES), Analytical and Reporting Guidelines, Hyattsville, Md, USA, 2006, http://www.cdc.gov/ nchs/nhanes/nhanes2003-2004/analytical_guidelines.htm.

[11] R. N. Forthofer, E. S. Lee, and M. Hernandez, Contingency Table Analysis. Biostatistics: A Guide to Design, Analysis and Discovery, Academic Press, Elsevier, Burlington, Mass, USA, 2nd edition, 2007.

[12] Center for Disease Control and Prevention (CDC), "Translating Research into Action for Diabetes, (TRIAD) fact sheet," Program Announcement Number 04005 and 98086 from the Centers for Disease Control and Prevention (CDC) Division of Diabetes Translation and the National Institute of Diabetes and Digestive and Kidney Diseases (NIDDK), 2009, http:// www.cdc.gov/diabetes/projects/pdfs/TRIAD_fact_sheet.pdf.

[13] Agency for Healthcare Research and Quality, 2008 National Healthcare Disparities Report (NHDR), Agency for Healthcare Research and Quality, Rockville, Md, USA, 2009.

[14] S. K. Vanderbilt, M. K. Wynia, M. Gadon, and G. C. Alexander, "A qualitative study of physicians' engagement in reducing healthcare disparities," Journal of the National Medical Association, vol. 99, no. 12, pp. 1315-1322, 2007.

[15] J. D. Piette, D. Schillinger, M. B. Potter, and M. Heisler, "Dimensions of patient-provider communication and diabetes self-care in an ethnically diverse population," Journal of General Internal Medicine, vol. 18, no. 8, pp. 624-633, 2003.

[16] R. BeLue, S. T. Lanza, and M. K. Figaro, "Lifestyle therapy changes and hypercholesterolemia: identifying risk groups in a community sample of blacks and whites," Ethnicity and Disease, vol. 19, no. 2, pp. 142-147, 2009.

[17] N. V. Oster, V. Welch, L. Schild, J. A. Gazmararian, K. Rask, and C. Spettell, "Differences in self-management behaviors and use of preventive services among diabetes management enrollees by race and ethnicity," Disease Management, vol. 9, no. 3, pp. 167-175, 2006.

[18] C. Nwasuruba, M. Khan, and L. E. Egede, "Racial/ethnic differences in multiple self-care behaviors in adults with diabetes," Journal of General Internal Medicine, vol. 22, no. 1, pp. 115-120, 2007.

[19] E. Brunner, I. White, M. Thorogood, A. Bristow, D. Curle, and M. Marmot, "Can dietary interventions change diet and 
cardiovascular risk factors? A meta-analysis of randomized controlled trials," American Journal of Public Health, vol. 87, no. 9, pp. 1415-1422, 1997.

[20] E. J. Brunner, K. Rees, K. Ward, M. Burke, and M. Thorogood, "Dietary advice for reducing cardiovascular risk," Cochrane Database of Systematic Reviews, no. 4, Article ID CD002128, 2007.

[21] J. L. Tang, J. M. Armitage, T. Lancaster, C. A. Silagy, G. H. Fowler, and H. A. W. Neil, "Systematic review of dietary intervention trials to lower blood total cholesterol in freeliving subjects," BMJ, vol. 316, no. 7139, pp. 1213-1219, 1998.

[22] M. R. Law, N. J. Wald, and S. G. Thompson, "By how much and how quickly does reduction in serum cholesterol concentration lower risk of ischaemic heart disease?" BMJ, vol. 308, no. 6925, pp. 367-372, 1994.

[23] C. Lee, S. L. Ayers, and J. J. Kronenfeld, "The association between perceived provider discrimination, healthcare utilization and health status in racial and ethnic minorities," Ethnicity and Disease, vol. 19, no. 3, pp. 330-337, 2009.

[24] A. Jerant, R. Arellanes, and P. Franks, "Health status among us hispanics: ethnic variation, nativity, and language moderation," Medical Care, vol. 46, no. 7, pp. 709-717, 2008.

[25] L. S. Wallace, J. E. Devoe, E. S. Rogers, J. Protheroe, G. Rowlands, and G. E. Fryer Jr., "Digging deeper: quality of patient-provider communication across Hispanic subgroups," BMC Health Services Research, vol. 9, article 240, 2009.

[26] J. Scholl, "Traditional dietary recommendations for the prevention of cardiovascular disease: do they meet the needs of our patients?" Cholesterol, vol. 2012, Article ID 367898, 9 pages, 2012.

[27] D.-C. Lee, X. Sui, T. S. Church, C. J. Lavie, A. S. Jackson, and S. N. Blair, "Changes in fitness and fatness on the development of cardiovascular disease risk factors: hypertension, metabolic syndrome, and hypercholesterolemia," Journal of the American College of Cardiology, vol. 59, no. 7, pp. 665-672, 2012. 


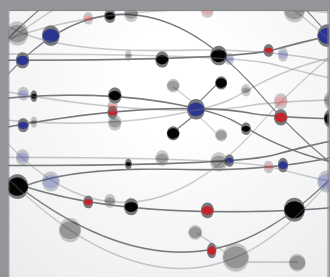

The Scientific World Journal
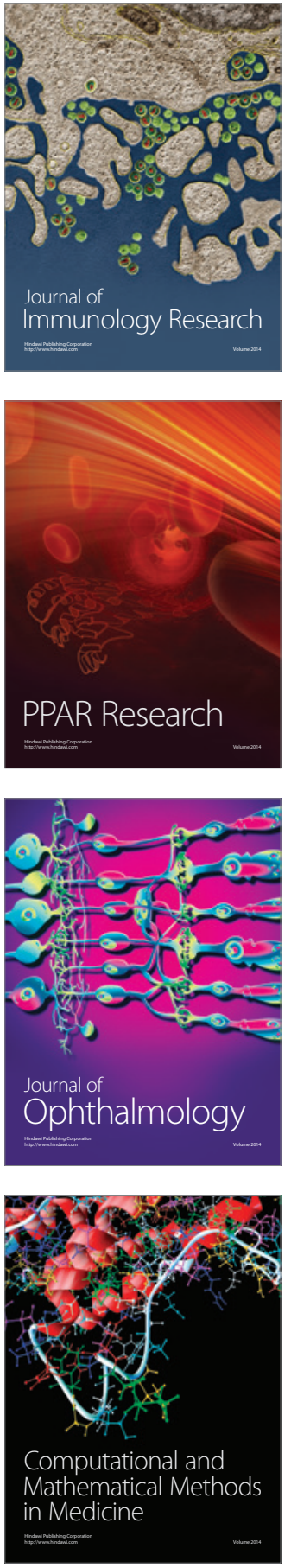

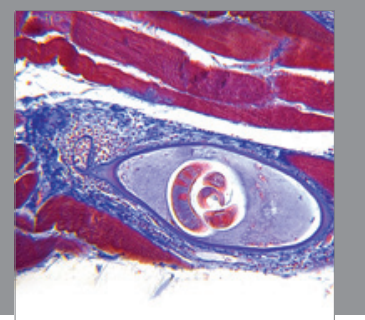

Gastroenterology

Research and Practice
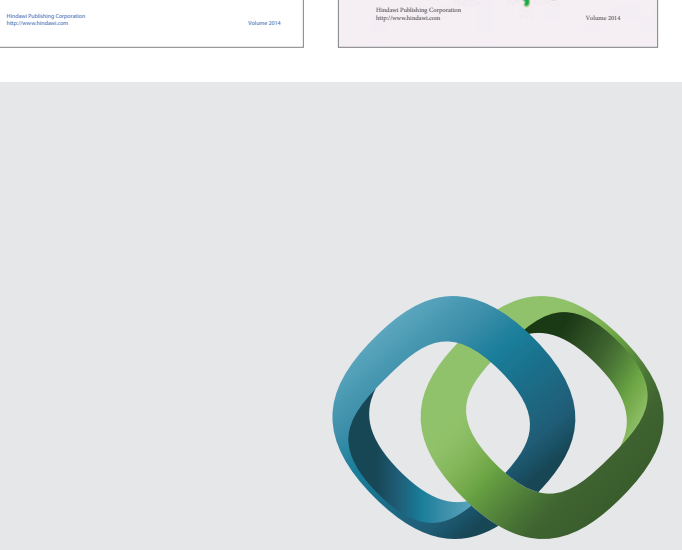

\section{Hindawi}

Submit your manuscripts at

http://www.hindawi.com
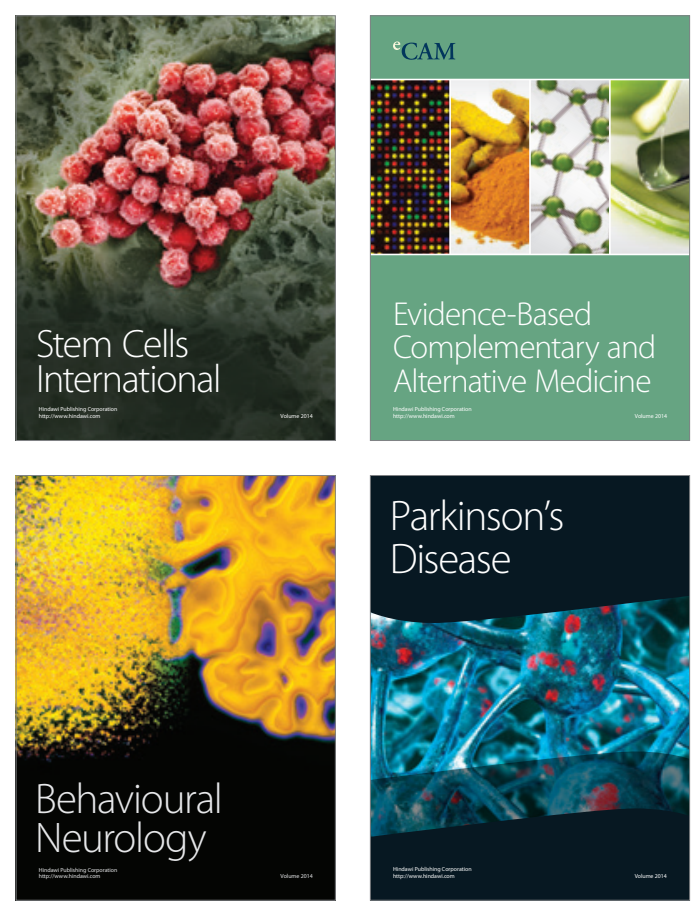

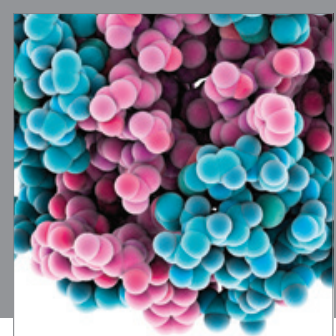

Journal of
Diabetes Research

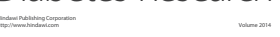

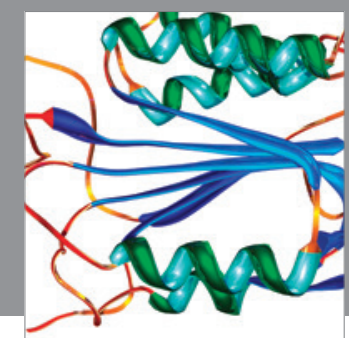

Disease Markers
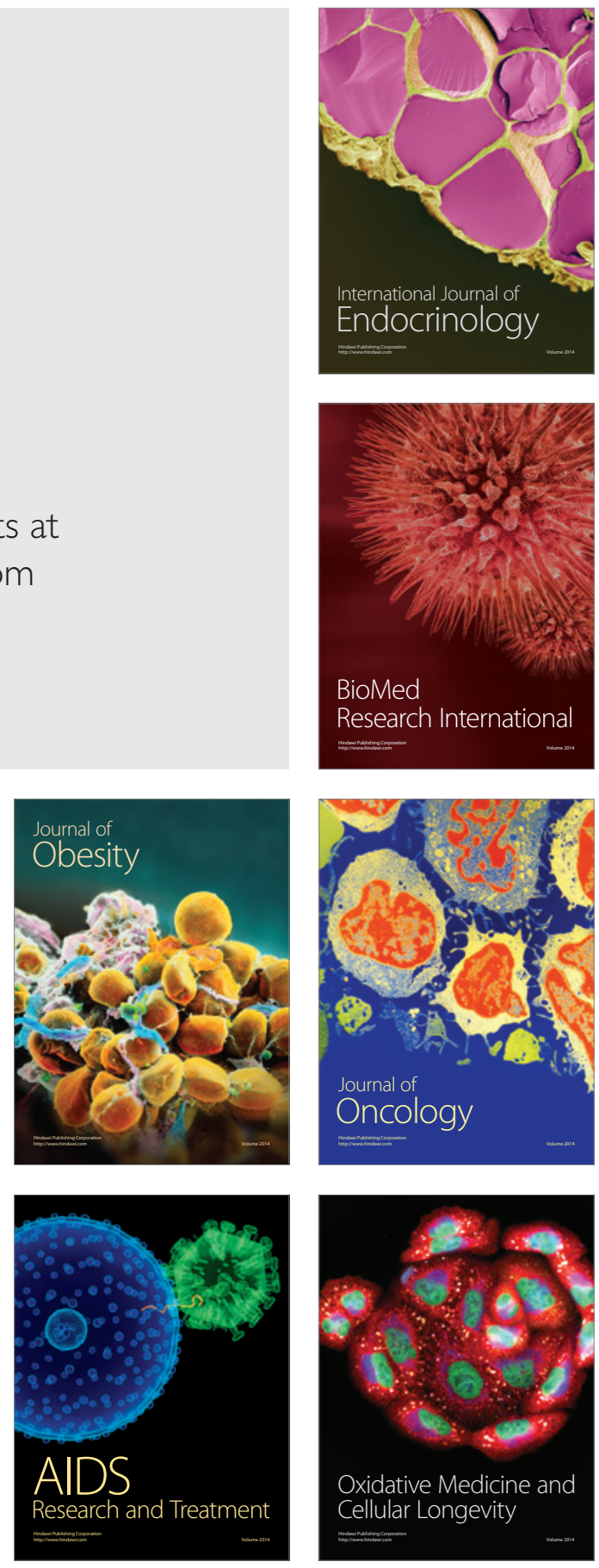